\title{
Progress in the Pathologic Diagnosis of Borderline Follicular Thyroid Tumor
}

\author{
Minghan Yang1,2†, Yaming Ji1, 2†, Jinku Zhang1, 2* \\ ${ }^{1}$ Key Laboratory of Molecular Pathology and Early Diagnosis of Tumor in Hebei Province, Baoding 071000, Hebei Province, \\ China \\ 2Pathology Department of Baoding First Central Hospital of Hebei Province, Baoding 071000, Hebei Province, China \\ ${ }^{\dagger}$ These authors contributed equally to this work \\ *Corresponding author: Jinku Zhang, 843561234@qq.com

\begin{abstract}
With the progress of science and technology as well as the development of ultrasound technology, more and more thyroid tumors have been found. Follicular tumor is one of the most common thyroid tumors, but borderline follicular tumors are relatively rare. At present, the diagnosis of borderline follicular thyroid tumor is unclear prior to surgery, and it is difficult to identify in frozen section or even conventional section. In order to effectively improve the diagnostic sensitivity and specificity of borderline follicular thyroid tumor, this paper summarizes the new WHO (World Health Organization) classification of borderline follicular thyroid tumor along with diagnostic methods, including clinical fine needle aspiration cytology, histopathology, and molecular biology, and reviews the research progress.
\end{abstract}

Keywords: Thyroid gland; Borderline tumor; Follicular tumor; Pathologic diagnosis

Publication date: September 2021; Online publication: September 30, 2021

\section{Introduction}

Thyroid follicular tumor is a tumor comprising of differentiated follicular cells. The new WHO Classification of Tumors of Endocrine Organs (2017) puts forward a new classification and diagnostic standard for thyroid follicular tumor. It can be divided into follicular thyroid adenoma (FTA), borderline follicular tumor, and follicular thyroid carcinoma (FTC) ${ }^{[1]}$. Borderline follicular tumor is a new type of tumor, which includes three subtypes: follicular tumor of undetermined malignant potential (FT-UMP), well-differentiated tumor of undetermined malignant potential (WT-UMP), and non-invasive follicular tumor with papillary-like nuclear features (NIFTP). The new classification not only improves the spectrum of thyroid diseases, but also defines the diagnostic criteria of borderline tumors, which provides a certain diagnostic basis for pathologists.

In practical, pathologic diagnosis is usually made based on cytology, histology, and molecular. Cytopathologic diagnosis is mainly based on the abnormal conditions in cells in order to study the causes of diseases, pathogeneses, and the laws of changes in the physiological function of cells in the disease process so as to put forward a basis for diagnosing and preventing diseases. Fine needle aspiration (FNA), as the most commonly used examination method, is not only convenient and barely traumatic, but also has a diagnostic accuracy of up to $90 \%{ }^{[2]}$. Its diagnostic value has been widely recognized ${ }^{[3]}$. However, due to limited FNA materials, it is impossible to observe the infiltration of tumors into blood vessels or capsules. Therefore, there are certain limitations in differentiating benign from malignant thyroid follicular tumors, thus still requiring histology for diagnosis ${ }^{[4]}$. Histopathologic diagnosis involves the derivation of a pathological tissue from a living body by local resection, forceps, removal, or other surgical methods; then, 
fixing the tissue in $10 \%$ formalin solution, and subsequently embedding with paraffin to form pathological sections. Thereafter, pathologic diagnosis can be made under the microscope. In addition to histopathologic diagnosis, molecular pathological examination, including the use of molecular and genetic methods, can improve the diagnostic accuracy and predict the development and prognosis of a disease.

Although the early diagnosis of benign and malignant thyroid follicular tumors helps to reduce misdiagnosis or missed diagnosis, it is of great significance to guide the treatment plan and improve patients' prognosis ${ }^{[5]}$. However, according to the current research situation, the diagnosis of borderline follicular thyroid tumor is unclear prior to surgical resection, in which the final diagnosis is often based on conventional pathological sections. This paper reviews the pathologic diagnosis and research progress of borderline follicular tumor types, which are newly added in the updated version of WHO's classification.

\section{Cytopathology}

As one of the most important challenges in thyroid cytology, the diagnosis of a follicular tumor or suspicious follicular tumor has great uncertainty because its diagnosis largely depends on the invasion of the capsule and vessels, which can only be seen in routine tissue sections. Although most of the follicular tumor specimens are highly cellular, the diagnosis cannot be made only by the number of cells. If follicular epithelial cells present as fragments of different sizes without any overlap or crowding, it can be considered as benign. If there is nuclear atypia, it cannot be diagnosed as malignant tumor or even a tumor because the enlargement or deep staining of the nucleus can also appear in thyroid hyperplasia nodules or follicular adenomas. At the same time, if the nuclear characteristics of papillary carcinoma are unclear or the structural characteristics of a classical papillary carcinoma are lacking, NIFTP, WT-UMP, and follicular subtype papillary carcinoma should be considered. In Zeng Su's review of the latest progress of NIFTPrelated clinical diagnosis, it has been pointed out that the main cytological features of NIFTP are microfiltration vesicle structures and papillary nucleus, including nuclear enlargement, nuclear crowding, irregular outlines, and obvious chromatin. Due to its lack of complete papillary structure and sand bodies, intranuclear inclusion bodies are also rare. This can be used as a differentiating characteristic from classical papillary thyroid carcinoma cells to a certain extent ${ }^{[6]}$.

\section{Histopathology}

\subsection{General observation}

In general, borderline follicular tumors are difficult to differentiate from FTA by naked eyes. Under naked eyes, tumors generally manifest as solid nodules with complete capsule, thin capsule, or no obvious capsule; the sizes of the tumors are different; the sections can be gray-white or gray-red; bleeding and necrosis are rare findings ${ }^{[7]}$. It is still necessary to observe the infiltration of tumor into vessels or capsule under the microscope to differentiate it from FTA. Some studies have shown that for encapsulated thyroid follicular tumors, some follicular adenomas can be upgraded to borderline follicular tumors, while some borderline follicular tumors can also be upgraded to follicular carcinoma via total removal or continuous resection of the capsule ${ }^{[8]}$. Therefore, under certain conditions, it may be necessary to obtain complete tumor materials.

\subsection{Microscopic features}

Borderline follicular tumors have intact capsules or clear boundaries with surrounding tissues. Their growth patterns are typical follicular-like structures. Atypical papillary structure and abundant cells can also be seen in the focal area. In addition, there are light to moderate heteromorphic nuclei without any sand bodies, tumor necrosis, or high mitotic activity. The difference is that the thyroid tumors with undetermined malignant potential (including FT-UMP and WDT-UMP) have suspicious capsules or vascular invasion. 
WDT-UMP has papillary carcinomatous nuclear features while FT-UMP does not have papillary carcinomatous nuclear features and although NIFTP has the characteristics of papillary carcinomatous nucleus, it has no suspicious capsule or vascular invasion ${ }^{[9]}$. When tumor cells infiltrate the fibrous capsule but do not penetrate it or when the fibrous capsule is mushroom-like or dome-like, capsule invasion is suspected. When tumor cells touch the blood vessels or nest in the vascular space that lacks endothelial cells, early vascular invasion is suspected. It is worth noting that in practical, there are some deviations or disputes about the understanding of capsules and vascular invasion ${ }^{[10]}$.

\subsection{Immunohistochemical expression}

Some scholars have detected the expression and significance of cytokeratin 19 (CK19), galectin-3, Hector battifora mesothelial cell-1 (HBME-1), and thyroid peroxidase (TPO) in WT-UMP by using the immunohistochemical method. They believe that CK19, galectin-3, HBME-1, and TPO can be used as potential markers to differentiate WT-UMP from benign thyroid lesions ${ }^{[11]}$. In order to screen and identify differentially expressed proteins of WT-UMP and to look for new protein markers, five differentially expressed proteins located in the cytoplasm of WT-UMP have been identified by immunohistochemistry: cytosolic non-specific dipeptidase (CNDP2), cytokeratin 18 (CK18), cathepsin B (CTSB), calreticulin (CRT), and annexin A5 (ANXA5). Moreover, the expressions of CK18, CTSB, CRT, and ANXA5 in WTUMP were found to be higher compared to those in normal thyroid. In addition, their expressions in papillary thyroid carcinoma were also higher than those found in WT-UMP, indicating that these five proteins may play a part in the occurrence and development of WT-UMP. This understanding is conducive to the early diagnosis of WT-UMP ${ }^{[12]}$. Shou Yiyi and other researchers studied 42 cases of welldifferentiated tumors with undetermined malignant potential by immunohistochemistry. The results showed that galectin-3 and HBME-1 were expressed in different degrees, suggesting that there is a certain correlation between these tumors and papillary carcinoma in their pathological mechanisms ${ }^{[13]}$. However, there was no specific expression of NIFTP, thus its diagnostic value is limited. In addition, Ki-67 proliferation index was low. In another research, Zhang Shan and other researchers discussed and retrospectively analyzed the clinicopathological features, immunophenotype, and prognosis of 40 cases of borderline thyroid tumors; they found that the immunohistochemical expression of thyroid transcription factor (TTF)-1 was positive in borderline thyroid tumors along with partially positive galectin-3, cyclin D1, CD56 (a neural cell adhesion molecule), and CK19. In addition, Ki-67 proliferation index was less than $5 \%$. It is concluded that borderline thyroid tumor is a type of tumor with inert biological behavior tendency, which is more common in women, having better prognosis and usually without invasion, metastasis, or recurrence $^{[14]}$.

\section{Molecular pathologic diagnosis}

Papotti and other scholars proposed to understand borderline lesions according to morphology combined with molecular biology ${ }^{[15]}$. Wang Yichao and others summarized the molecular research progress of NIFTP, and concluded that the most common molecular mutation in NIFTP is the rat sarcoma virus oncogene homolog (RAS) and the expression of Raf kinase protein can be seen $\beta$. In general, there was no BRAF V600E mutation in isomer K601E (B-type Raf, BRAF K601E), peroxisome proliferator-activated receptor gamma (PPARG) fusion, and thyroid adenoma-associated (THADA) fusion. They suggested that the abnormal expression of microRNAs also exists in NIFTP, and the molecules related to NIFTP may become new molecular markers for clinical diagnosis ${ }^{[16]}$. Similarly, Zhao Ru Nan and other scholars also supported this view in the study of NIFTP, where BRAF V600E was also negative in patients with NITFP [17]. However, some studies have reported that about $7 \%$ to $29 \%$ of NIFTP patients have BRAF V600E 
mutations ${ }^{[18,19]}$. While researching about NITFP, Han Mei and other scholars found no mutation in KRAS, NRAS, and BRAF genes ${ }^{[20]}$. Yang Ningning and other researchers pointed out that the detection of Ras gene mutation is helpful in the diagnosis of NITFP ${ }^{[21]}$. Hofman and other researchers studied a series of molecular mutations and chromosome rearrangements in tumors with undetermined malignant potential. The results showed that there were no BRAFV600E, RET/PTC-1, RET/PTC-3, and PAX8/PPAR mutations

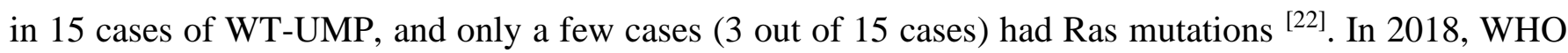
included molecular testing into the diagnostic criteria, suggesting the importance of molecular testing.

\section{Conclusion}

In conclusion, for borderline follicular thyroid tumors, it is difficult to diagnose only by cytopathology; the diagnosis is mainly based on histopathology and auxiliary molecular pathology. Fine needle aspiration combined with paraffin section, immunohistochemistry, and molecular pathology has high application value in the diagnosis of thyroid borderline follicular tumor. These methods can effectively improve the diagnostic sensitivity and specificity. For borderline follicular thyroid tumor, due to its inert biological behavior, the treatment is generally by simple thyroid lobectomy, and no longer using radioactive iodine; its prognosis is good with few recurrences and metastases ${ }^{[23]}$. This paper summarizes the pathological characteristics of this type of tumor, which is helpful for pathologists and clinicians to improve their understanding and the key points in diagnosing the disease. It lays a good foundation for the clinical diagnosis of the disease and provide an important basis for reasonable clinical treatment.

\section{Disclosure statement}

The authors declare that there is no conflict of interest.

\section{References}

[1] Rusinek D, Chmielik E, Krajewska J, et al., 2017, Current Advances in Thyroid Cancer Management - Are We Ready for the Epidemic Rise of Diagnoses?. Int J Mol Sci, 18(8).

[2] Li Y, 2010, Application Value of Ultrasound-Guided Biopsy in the Diagnosis of Nodular Goiter with Thyroid Cancer. Jilin Medical Journal, 31(23): 3871-3872.

[3] Liu Z, Zhou G, Kennichi K, 2016, Biological Behavior of Thyroid Cancer in Thyroid Fine Needle Aspiration with Uncertain Types of Lesions. Chinese Journal of Pathology, 45(6): 423-426.

[4] Daniels GH, 2018, Follicular Thyroid Carcinoma: A Perspective. Thyroid, 28(10): 1229-1242.

[5] Chen Y, 2020, Clinical Study on Diagnosis and Treatment of Thyroid Follicular Tumor. Jilin University, 42(6): 530-534.

[6] Zeng S, Xu P, Guo M, 2020, Clinical Research Progress of Non-Invasive Follicular Thyroid Tumor with Papillary Nucleus. Journal of Shanghai Jiaotong University (Medical Edition), 40(7): 969-973, 968.

[7] Gao M, Wei S, 2019, Progress in Diagnosis and Treatment of Non-Invasive Follicular Thyroid Tumor with Papillary Nucleus. Cancer Prevention and Treatment, 32(12): 1039-1044.

[8] Hamza A, Khawar S, Alrajjal A, et al., 2019, Processing the Entire Capsule in Thyroid Follicular Lesions Improves Diagnostic Accuracy. Annals of Diagnostic Pathology, 39: 21-24.

[9] Fang S, Xiao H, Nikiforov YE, et al., 2019, Changes of Diagnostic Criteria for Non-Invasive Follicular Thyroid Tumor with Papillary Nucleus Features. J Clin Exp Pathol, 35(1): 55. 
[10] Lei Y, Pan Y, Yang T, et al., 2019, Recognition and Retrospective Analysis of Borderline Thyroid Tumor. Journal of Clinical and Experimental Pathology, 35(8): 968-971.

[11] Feng Y, Zhang L, Li Q, et al., 2017, Expression and Significance of CK19, Galectin-3, HBME-1 and TPO in Well Differentiated Thyroid Tumors with Undetermined Malignant Potential. Journal of Practical Oncology, 32(4): 362-366.

[12] Tang M, Yang H, Liu P, et al., 2018, Proteomic Analysis of Differentially Expressed Proteins in Well Differentiated Thyroid Tumors with Undetermined Malignant Potential. China Medical Journal, 15(7): 60-65, 181.

[13] Shou YY, Cai LB, Chen LJ, et al., 2012, Expression and Clinical Significance of Galectin-3 and HBME-1 in Well Differentiated Thyroid Tumors with Undetermined Malignant Potential and Follicular Structure. Zhejiang Practical Medicine, 17(4): 243-244, 255.

[14] Zhang S, Feng Z, Yang N, et al., 2020, Clinicopathological Characteristics and Prognosis of 40 Cases of Borderline Thyroid Tumor. Journal of Clinical and Experimental Pathology, 36(10): 1202-1204, 1208.

[15] Papotti M, Rodriguez J, De Pompa R, et al., 2005, Galectin-3 and HBME-1 Expression in WellDifferentiated Thyroid Tumors with Follicular Architecture of Uncertain Malignant Potential. Mod Pathol, 18(4): 541-546.

[16] Wang Y, Xiang L, Zhou P, et al., 2019, Molecular Research Progress of Non-Invasive Follicular Thyroid Tumor with Papillary Carcinomatous Nucleus. Chinese Journal of Basic and Clinical General Surgery, 26(1): 110-115.

[17] Zhao RN, Liu Y, He M, et al., 2020, Clinicopathological Analysis of 6 Cases of Thyroid Non-Invasive Follicular Tumor with Papillary Carcinomatous Nucleus. Journal of Clinical and Experimental Pathology, 36(5): 574-576.

[18] Kim TH, Lee M, Kwon AY, 2018, Molecular Genotyping of the Non-Invasive Encapsulated Follicular Variant of Papillary Thyroid Carcinoma. Histopathology, 72(4): 648-661.

[19] Johnson DN, Sadow PM, 2018, Exploration of BRAFV600E as a Diagnostic Adjuvant in the NonInvasive Follicular Thyroid Neoplasm with Papillary-Like Nuclear Features (NIFTP). Hum Pathol, 82: 32-38.

[20] Han M, He Y, Wang Y, et al., 2018, Clinicopathological Analysis of 4 Cases of Non-Invasive Follicular Thyroid Tumor with Papillary Nucleus. Journal of Diagnostic Pathology, 25(11): 750-753.

[21] Yang N, Huang Y, Yan W, et al., 2020, Clinicopathological Characteristics of Non-Invasive Follicular Thyroid Tumor with Papillary Nucleus. Chinese Journal of Oncology, 47(17): 886-889.

[22] Hofman V, Lassalle S, Bonnetaud C, et al., 2009, Thyroid Tumours of Uncertain Malignant Potential: Frequency and Diagnostic Reproducibility. Virchows Arch, 455(1): 21-33.

[23] Zhang Z, Chen T, Yang C, et al., 2019, Clinicopathology of Thyroid Tumor with Undetermined Malignant Potential. Journal of Kunming Medical University, 40(9): 83-88. 